\title{
Mechanical Design of AM Fabricated Prismatic Rods under Torsion
}

\author{
Alexander V. Manzhirov ${ }^{1,2,3,4}$ \\ ${ }^{1}$ Institute for Problems in Mechanics of the Russian Academy of Sciences, Vernadsky Ave 101 Bldg 1, Moscow, 119526, Russia \\ ${ }^{2}$ Bauman Moscow State Technical University, 2nd Baumanskaya Str 5/1, Moscow, 105005, Russia \\ ${ }^{3}$ National Research Nuclear University T«MEPhITi, Kashirskoye shosse 31, Moscow, 115409, Russia \\ ${ }^{4}$ Moscow Technological University, Vernadsky Ave 78, Moscow, 119454, Russia
}

\begin{abstract}
We study the stress-strain state of viscoelastic prismatic rods fabricated or repaired by additive manufacturing technologies under torsion. An adequate description of the processes involved is given by methods of a new scientific field, mechanics of growing solids. Three main stages of the deformation process (before the beginning of growth, in the course of growth, and after the termination of growth) are studied. Two versions of statement of two problems are given: (i) given the torque, find the stresses, displacements, and torsion; (ii) given the torsion, find the stresses, displacements, and torque. Solution methods using techniques of complex analysis are presented. The results can be used in mechanical and instrument engineering.
\end{abstract}

\section{Statement of the torsion problem}

Consider a homogeneous viscoelastic ageing rod manufactured at the initial instant of time and occupying some cylindrical domain $\Pi_{1}$. The lateral surface $\Pi_{1}$ of the body is stress-free until time $\tau_{0} \geq 0$ at which forces statically equivalent to a couple with torque $M(t)$ are applied to the faces of the cylindrical body.

At time $\tau_{1} \geq \tau_{0}$, continuous accretion of the rod by elements manufactured simultaneously with the rod begins. Let $L(t)$ be the time-varying boundary of the cross-section $\Omega(t)$, so that $L\left(\tau_{1}\right)=L_{1}$ and $\Omega\left(\tau_{1}\right)=\Omega_{1}$. The boundary $L(t)$ consists of two parts, $L(t)=L^{*}(t) \cup L_{\sigma}(t)$. Here $L^{*}(t)$ is the accretion boundary (or the growth boundary) on which the material influx occurs at time $t$, and $L^{*}(t)=L^{*}$ for $\tau<\tau_{1} ; L_{\sigma}(t)$ is the stress-free boundary. We also assume that the time $\tau_{0}\left(x_{1}, x_{2}\right)$ at which the load is applied to the elements being added coincides with the time $\tau^{*}\left(x_{1}, x_{2}\right)$ at which they are added to the growing rod.

The accretion is terminated at time $\tau_{2} \geq \tau_{1}$, and since then the rod occupies some domain $\Pi_{2}=\Pi\left(\tau_{2}\right)$ whose cross-section $\Omega_{2}=\Omega\left(\tau_{2}\right)$ has the boundary $L_{2}=L\left(\tau_{2}\right)$. The boundary conditions posed on the boundary $L_{2}=L\left(\tau_{2}\right)$ are the same as on the boundary $L_{1}$ before the beginning of growth.

Somewhat later, at time $\tau_{3} \geq \tau_{2}$, accretion may begin again; it may well happen that the new growth boundary is not related in any way to the earlier existing one. Then one can assume that accretion terminates at time $\tau_{4}$, etc., thus successively arriving at the problem of piecewise continuous accretion of a solid rod with $n$ growth beginning instants and accordingly $n$ growth termination instants.

We proceed to the analysis of main stages of the piecewise continuous accretion process for viscoelastic rods. From now on, we consider sufficiently slow processes, and so inertial terms in the equilibrium equations can be neglected (also see [1-7]).

\section{Boundary value problem for the rod of fixed composition}

Consider the stress-strain state of a viscoelastic ageing rod of fixed composition on the time interval $t \in\left[\tau_{0}, \tau_{1}\right]$. We have the boundary value problem (e.g., see [1-3]) consisting of the equilibrium equations

$$
\frac{\partial \sigma_{13}}{\partial x_{3}}=0, \quad \frac{\partial \sigma_{23}}{\partial x_{3}}=0, \quad \frac{\partial \sigma_{13}}{\partial x_{1}}+\frac{\partial \sigma_{23}}{\partial x_{2}}=0,
$$

the strain-displacement relations

$$
\varepsilon_{i j}=\frac{1}{2}\left(\frac{\partial u_{i}}{\partial x_{j}}+\frac{\partial u_{j}}{\partial x_{i}}\right)=0, \varepsilon_{i i}=\varepsilon_{12}=0, i, j=1,2,3,
$$

the boundary condition

$$
\left(x_{1}, x_{2}\right) \in L_{1}: \quad \sigma_{13} n_{1}+\sigma_{23} n_{2}=0 ;
$$

on the lateral surface, and the constitutive equation 


$$
\begin{gathered}
\sigma_{13}=2 G\left(I+N\left(\tau_{0}, t\right)\right) \varepsilon_{13}, \\
\sigma_{23}=2 G\left(I+N\left(\tau_{0}, t\right)\right) \varepsilon_{23}, \\
\left(I+N\left(\tau_{0}, t\right)\right)^{-1}=\left(I-L\left(\tau_{0}, t\right)\right), \\
L\left(\tau_{0}, t\right) f(t)=\int_{\tau_{0}}^{t} f(\tau) K_{1}(t, \tau) d \tau, \\
K_{1}(t, \tau)=G(\tau) \frac{\partial}{\partial \tau}\left[G^{-1}(\tau)+\omega(t, \tau)\right],
\end{gathered}
$$

where $\vec{n}=\left\{n_{1}, n_{2}\right\}$ is the unit outward normal vector on the lateral surface of the body, $G=G(t)$ is the instantaneous elastic shear modulus, $K_{1}(t, \tau)$ and $\omega(t, \tau)$ are the shear creep kernel and the shear creep rate kernel, respectively, and $I$ is the identity operator. The arguments have been omitted above in a number of obvious cases. We also omit them in what follows unless their absence can lead to a misunderstanding.

Further, the following equilibrium conditions should hold for the end cross-section $\Omega_{1}$ subjected to the torque $M(t)$ :

$$
\begin{gathered}
M(t)=\iint_{\Omega_{1}}\left(x_{1} \sigma_{23}-x_{2} \sigma_{13}\right) d x_{1} d x_{2} \\
\iint_{\Omega_{1}} \sigma_{13} d x_{1} d x_{2}=0, \quad \iint_{\Omega_{1}} \sigma_{23} d x_{1} d x_{2}=0 .
\end{gathered}
$$

Relations (1)-(5) form a problem of the viscoelasticity theory for a homogeneous ageing body. The solution of this problem permits one to describe the stress-strain state of the body on the time interval $t \in\left[\tau_{0}, \tau_{1}\right]$.

Let us transform the boundary value problem for the main body. To this end, we divide the expressions containing the stresses $\sigma_{13}$ and $\sigma_{23}$ in (1)-(5) by G and apply the operator $\left(I-L\left(\tau_{0}, t\right)\right)$ to them. Since the operator $\left(I-L\left(\tau_{0}, t\right)\right)$ commutes with the divergence operator, we see that the expressions (1)-(4) acquire the form

$$
\begin{gathered}
\frac{\partial \sigma_{13}^{0}}{\partial x_{3}}=0, \quad \frac{\partial \sigma_{23}^{0}}{\partial x_{1}}=0, \quad \frac{\partial \sigma_{13}^{0}}{\partial x_{1}}+\frac{\partial \sigma_{23}^{0}}{\partial x_{2}}=0, \\
\varepsilon_{i i}=\frac{\partial u_{i}}{\partial x_{i}}=0, \varepsilon_{i j}=\frac{1}{2}\left(\frac{\partial u_{i}}{\partial x_{j}}+\frac{\partial u_{j}}{\partial x_{i}}\right)=0, i, j=1,2,3, \\
\left(x_{1}, x_{2}\right) \in L_{1}: \quad \sigma_{13}^{0} n_{1}+\sigma_{23}^{0} n_{2}=0 \\
\sigma_{13}^{0}=2 \varepsilon_{13}, \quad \sigma_{23}^{0}=2 \varepsilon_{23},
\end{gathered}
$$

where $\sigma_{i j}^{0}$ and $\sigma_{i j}$ are related by the formula

$$
\sigma_{i j}^{0}=\left(I-L\left(\tau_{0}, t\right)\right) \sigma_{i j} G^{-1} .
$$

Likewise, relations (5) become

$$
\begin{aligned}
& M^{0}(t)=\iint_{\Omega_{1}}\left(x_{1} \sigma_{23}^{0}-x_{2} \sigma_{13}^{0}\right) d x_{1} d x_{2}, \\
& \iint_{\Omega_{1}} \sigma_{13}^{0} d x_{1} d x_{2}=0, \quad \iint_{\Omega_{1}} \sigma_{23}^{0} d x_{1} d x_{2}=0,
\end{aligned}
$$

where $M^{0}=\left(I-L\left(\tau_{0}, t\right)\right) M G^{-1}$.
The boundary value problem (6)-(8), in contrast to problem (1)-(5), contains time as a parameter and is mathematically equivalent to a boundary value problem of the elasticity theory with parameter $t$.

By substituting the strain-displacement relations into the equilibrium equations of problem (6), we obtain the equilibrium equations in terms of displacements in the form

$$
\begin{aligned}
& \frac{\partial^{2} u_{1}}{\partial x_{3}^{2}}+\frac{\partial^{2} u_{3}}{\partial x_{3} \partial x_{1}}=0, \quad \frac{\partial^{2} u_{2}}{\partial x_{3}^{2}}+\frac{\partial^{2} u_{3}}{\partial x_{3} \partial x_{2}}=0, \\
& \frac{\partial^{2} u_{1}}{\partial x_{3} \partial x_{1}}+\frac{\partial^{2} u_{3}}{\partial x_{1}^{2}}+\frac{\partial^{2} u_{2}}{\partial x_{3} \partial x_{2}}+\frac{\partial^{2} u_{3}}{\partial x_{2}^{2}}=0 .
\end{aligned}
$$

We use the first three strain-displacement relations in (6) to obtain

$$
u_{i}=u_{i}\left(x_{2}, x_{3}, t\right), \quad i=1,2,3,
$$

where the displacement $u_{i}$ is independent of the coordinate $x_{i}$.

In view of (9) and (10), the equilibrium equations in terms of displacements become

$$
\frac{\partial^{2} u_{1}}{\partial x_{3}^{2}}=0, \quad \frac{\partial^{2} u_{2}}{\partial x_{3}^{2}}=0, \quad \frac{\partial^{2} u_{3}}{\partial x_{1}^{2}}+\frac{\partial^{2} u_{3}}{\partial x_{2}^{2}}=0 .
$$

It follows from the third equation in (11) that the function $u_{3}\left(x_{1}, x_{3}, t\right)$ is harmonic. Let us find $u_{1}\left(x_{2}, x_{3}, t\right)$ and $u_{2}\left(x_{1}, x_{3}, t\right)$. To this end, we integrate the first two equations in (11) twice with respect to $x_{3}$. As a result, we obtain

$$
\begin{aligned}
& u_{1}=f\left(x_{2}, t\right) x_{3}+c_{1}(t) x_{3}+f_{1}\left(x_{2}, t\right)+c_{3}(t), \\
& u_{2}=g\left(x_{1}, t\right) x_{3}+c_{2}(t) x_{3}+g_{1}\left(x_{2}, t\right)+c_{4}(t) .
\end{aligned}
$$

By using (12) and the fourth strain-displacement relation in (6), we obtain

$$
\frac{\partial f}{\partial x_{2}} x_{3}+\frac{\partial f_{1}}{\partial x_{2}}+\frac{\partial g}{\partial x_{1}} x_{3}+\frac{\partial g_{1}}{\partial x_{1}}=0 .
$$

Equation (13) permits us to conclude that

$$
g=\theta(t) x_{1}, f=-\theta(t) x_{2}, g_{1}=c(t) x_{1}, f_{1}=-c(t) x_{2} \text {. }
$$

By (14), for the displacements $u_{1}$ and $u_{2}$ we obtain

$$
\begin{aligned}
& u_{1}=-\theta(t) x_{2} x_{3}+c_{1}(t) x_{3}-c(t) x_{2}+c_{3}(t), \\
& u_{2}=\theta(t) x_{1} x_{3}+c_{2}(t) x_{3}+c(t) x_{1}+c_{4}(t) .
\end{aligned}
$$

The last terms in the expression (15) do not affect the stress state of the twisted cylindrical body. To eliminate the translational displacements of the rod along the $x_{1}$ and $x_{2}$-axes, assume that some point of the rod end is fixed, so that $x_{1}=x_{2}=0$ at that point. We place the origin there and require that the additional conditions

$$
\frac{\partial u_{1}}{\partial x_{3}}=\frac{\partial u_{2}}{\partial x_{3}}=\frac{\partial u_{2}}{\partial x_{1}}=0
$$


hold at that point so as to prevent the rigid rotations of the cylindrical body about the $x_{1}-, x_{2}-$, and $x_{3}$-axes. Then $c(t)=c_{1}(t)=c_{2}(t)=c_{3}(t)=c_{4}(t)=0$, and hence the expressions (15) for the stresses $x_{1}$ and $x_{2}$ acquire the form

$$
u_{1}=-\theta(t) x_{2} x_{3}, \quad u_{2}=\theta(t) x_{3} x_{1} .
$$

The function $\theta(t)$ is called the torsion angle (or torsion) and is related to the displacements by the formula

$$
\frac{\partial}{\partial x_{3}}\left(\frac{\partial u_{1}}{\partial x_{2}}-\frac{\partial u_{2}}{\partial x_{1}}\right)=-2 \theta(t) .
$$

By analogy with $[8]$, set

$$
u_{3}=\theta(t) \varphi_{\tau_{1}}
$$

where $\varphi_{\tau_{1}}$ is some function to be determined of the variables $x_{1}, x_{2}$, and $\tau_{1}$. It is called the torsion function and characterizes the deformation of cross-sections.

Then, by (16) and (18), we have

$$
u_{1}=-\theta(t) x_{2} x_{3}, \quad u_{2}=\theta(t) x_{3} x_{1}, \quad u_{3}=\theta(t) \varphi_{\tau_{1}} .
$$

By using relations (19) and the constitutive equations in (6), we obtain

$$
\sigma_{13}^{0}=\theta(t)\left(\frac{\partial \varphi_{\tau_{1}}}{\partial x_{1}}-x_{2}\right), \quad \sigma_{23}^{0}=\theta(t)\left(\frac{\partial \varphi_{\tau_{1}}}{\partial x_{2}}+x_{1}\right)
$$

By substituting (20) into the third equilibrium equation in (3), we find that the function $\varphi_{\tau_{1}}$ satisfies the 2D Laplace equation

$$
\frac{\partial^{2} \varphi_{\tau_{1}}}{\partial x_{1}^{2}}+\frac{\partial^{2} \varphi_{\tau_{1}}}{\partial x_{2}^{2}}=0
$$

i.e., the torsion function $\varphi_{\tau_{1}}$ is harmonic in the domain $\Omega_{1}$.

Let us find the boundary condition for the function $\varphi_{\tau_{1}}$ on the contour $L_{1}$ of the cross-section $\Omega_{1}$. By substituting (20) into the boundary condition (3), we obtain

$$
\left(x_{1}, x_{2}\right) \in L_{1}: \quad \frac{\partial \varphi_{\tau_{1}}}{\partial n}=x_{2} n_{1}-x_{1} n_{2}
$$

Thus, we have reduced the original torsion problem for a prismatic body to the Neumann problem for the function $\varphi_{\tau_{1}}$ in the domain $\Omega_{1}$, that is, the problem of finding a harmonic function $\varphi_{\tau_{1}}$ in the cross-section domain $\Omega_{1}$ (see (1.21)) from the given values of its normal derivative on the contour $L_{1}$ (see (1.22)).

Since

$$
x_{1} \sigma_{23}^{0}-x_{2} \sigma_{13}^{0}=\theta(t)\left(x_{1}^{2}+x_{2}^{2}+x_{1} \frac{\partial \varphi_{\tau_{1}}}{\partial x_{2}}-x_{2} \frac{\partial \varphi_{\tau_{1}}}{\partial x_{1}}\right),
$$

We see that the first integral equilibrium condition in (8) acquires the form

$$
\begin{aligned}
& M^{0}(t)=\left(I-L\left(\tau_{0}, t\right)\right) M(t) G^{-1}(t)=\theta(t) D_{1}, \\
& D_{1}=\iint_{\Omega_{1}}\left(x_{1}^{2}+x_{2}^{2}+x_{1} \frac{\partial \varphi_{\tau_{1}}}{\partial x_{2}}-x_{2} \frac{\partial \varphi_{\tau_{1}}}{\partial x_{1}}\right) d x_{1} d x_{2},
\end{aligned}
$$

and the second and third conditions are satisfied identically.

The constant $D_{1}$ is called the torsional rigidity. It only depends on the shape of the cross-section of the body.

The problem in question has two possible statements:

1. Given the torque $M(t)$, determine the stresses $\sigma_{i j}$, the displacements $u_{i}$, and the torsion angle $\theta(t)$.

2. Given the torsion angle $\theta(t)$, find $\sigma_{i j}, u_{i}$, and the torque $M(t)$.

The solution for the first statement can be constructed as follows: find the function $\varphi_{\tau_{1}}$ from the boundary condition, use formulas (24) to find $\theta(t)$, use formulas (19) and (20) to find $u_{i}$ and $\sigma_{i j}^{0}$, and finally reconstruct the true stresses $\sigma_{i j}$ by the formula

$$
\sigma_{i j}=G(t)\left(\sigma_{i j}^{0}++\int_{\tau_{0}}^{t} \sigma_{i j}^{0} R_{1}(t, \tau) d \tau\right),
$$

where $R_{1}(t, \tau)$ is the resolvent of the kernel $K_{1}(t, \tau)$.

For the second statement of the problem, one determines $\varphi_{\tau_{1}}$, finds $u_{i}$ and $\sigma_{i j}^{0}$ by formulas (19) and (20), reconstructs $\sigma_{i j}$ with the use of (25), and determines $M(t)$ on the basis of (5).

Sometimes it is convenient to study the problem for the main body in terms of rates of the corresponding variables, because information about these rates is needed when solving the problem at the growth stage. To this end, one should differentiate relations (6) with respect to $t$. (From now on, differentiation is always understood in the sense of distributions.) Then we have

$$
\begin{gathered}
\frac{\partial S_{13}}{\partial x_{3}}=0, \quad \frac{\partial S_{23}}{\partial x_{1}}=0, \quad \frac{\partial S_{13}}{\partial x_{1}}+\frac{\partial S_{23}}{\partial x_{2}}=0 \\
D_{i i}=\frac{\partial v_{i}}{\partial x_{i}}=0, \quad D_{i j}=\frac{1}{2}\left(\frac{\partial v_{i}}{\partial x_{j}}+\frac{\partial v_{j}}{\partial x_{i}}\right)=0, i, j=1,2,3, \\
\left(x_{1}, x_{2}\right) \in L_{1}: \quad S_{13} n_{1}+S_{23} n_{2}=0 \\
S_{13}=2 D_{13}, \quad S_{23}=2 D_{23}, \\
S_{i j}=\frac{\partial \sigma_{i j}^{0}}{\partial t}, \quad v_{i}=\frac{\partial u_{i}}{\partial t}, \quad D_{i j}=\frac{\partial \varepsilon_{i j}}{\partial t} .
\end{gathered}
$$

Relations (26) form an initial-boundary value problem parametrically depending on time $t$. 
By analogy with the preceding, one can show that $v_{i}$ and $S_{i j}$ have the following structure:

$$
\begin{aligned}
& v_{1}=-\theta^{\bullet}(t) x_{2} x_{3}, v_{2}=\theta^{\bullet}(t) x_{3} x_{1}, v_{3}=\theta^{\bullet}(t) \varphi_{\tau_{1}}, \\
& S_{13}=\theta^{\bullet}(t)\left(\frac{\partial \varphi_{\tau_{1}}}{\partial x_{1}}-x_{2}\right), S_{23}=\theta^{\bullet}(t)\left(\frac{\partial \varphi_{\tau_{1}}}{\partial x_{2}}+x_{1}\right),
\end{aligned}
$$

where the dot stands for differentiation with respect to $t$ (we use this notation in what follows) and $\theta^{\bullet}(t)$ is the torsion angular velocity.

By substituting (27) into the third equilibrium equation in (26), we find that the function $\varphi_{\tau_{1}}$ satisfies the 2D Laplace equation

$$
\frac{\partial^{2} \varphi_{\tau_{1}}}{\partial x_{1}^{2}}+\frac{\partial^{2} \varphi_{\tau_{1}}}{\partial x_{2}^{2}}=0
$$

i.e., the torsion function $\varphi_{\tau_{1}}$ is harmonic in the domain $\Omega_{1}$.

The boundary condition for the function $\varphi_{\tau_{1}}$ on the contour $L_{1}$ of the cross-section $\Omega_{1}$ acquires a form similar to (22),

$$
\left(x_{1}, x_{2}\right) \in L(t): \frac{\partial \varphi_{\tau_{1}}}{\partial n}=x_{2} n_{1}-x_{1} n_{2} .
$$

Thus, we have reduced the original torsion problem for a prismatic body to the Neumann problem for the function $\varphi_{\tau_{1}}$ in the domain $\Omega_{1}$.

The differentiated relation (24) becomes

$$
\begin{gathered}
M^{0 \bullet}(t)=\frac{M^{\bullet}(t)}{G(t)}+\int_{\tau_{0}}^{t} \frac{\partial M(t)}{\partial \tau} \frac{\partial \omega(t, \tau)}{\partial t} d \tau+M\left(\tau_{0}\right) \frac{\partial \omega\left(t, \tau_{0}\right)}{\partial t}, \\
M^{0 \bullet}(t)=\theta^{\bullet}(t) D_{1} .
\end{gathered}
$$

To solve the first version of the problem (where the torque $M(t)$ is given), one should use the boundary condition to find $\varphi_{\tau_{1}}$, then determine $v_{i}$ and $S_{i j}$ by formulas (28), use formulas (30) to find the torsion angular velocity, and reconstruct the true stresses, displacement, and torsion angle by the formulas

$$
\begin{gathered}
\sigma_{i j}\left(x_{1}, x_{2}, t\right)=G(t)\left[\frac{\sigma_{i j}\left(x_{1}, x_{2}, \tau_{0}\right)}{G\left(\tau_{0}\right)}\left(1+\int_{\tau_{0}}^{t} R(t, \tau) d \tau\right)+\right. \\
\left.+\int_{\tau_{0}}^{t}\left(S_{i j}\left(x_{1}, x_{2}, \tau\right)+\int_{\tau_{0}}^{\tau} S_{i j}\left(x_{1}, x_{2}, \varsigma\right) d \varsigma R(t, \tau)\right) d \tau\right] \\
u_{i}\left(x_{1}, x_{2}, t\right)=u_{i}\left(x_{1}, x_{2}, \tau_{0}\right)+\int_{\tau_{0}}^{t} v_{i}\left(x_{1}, x_{2}, \tau\right) d \tau \\
\theta(t)=\theta\left(\tau_{0}\right)+\int_{\tau_{0}}^{t} \theta^{\bullet}(\tau) d \tau .
\end{gathered}
$$

To solve the second version of the problem (where the torsion angle $\theta(t)$ is given), one should find $\varphi_{\tau_{1}}$, determine $v_{i}$ and $S_{i j}$ by formulas (28), reconstruct $u_{i}$ and $\sigma_{i j}$ by formulas (31), and find the torque according to $(5)$.

Note that the initial values of the stresses, displacements, and torsion angle in (31) are determined from the solution of the boundary value problem (1)-(5) or (6) for $t=\tau_{0}$.

Thus, the study of the torsion problem for a growing body at the stage preceding accretion is complete.

\section{Initial-boundary value problem for a growing rod}

Now consider the process of continuous accretion of a deformable body for $t \in\left[\tau_{1}, \tau_{2}\right]$. For a growing rod, we have the equilibrium equations

$$
\frac{\partial \sigma_{13}}{\partial x_{3}}=0, \quad \frac{\partial \sigma_{23}}{\partial x_{3}}=0, \quad \frac{\partial \sigma_{13}}{\partial x_{1}}+\frac{\partial \sigma_{23}}{\partial x_{2}}=0 ;
$$

the strain rate-displacement rate relations

$$
D_{i j}=\frac{1}{2}\left(\frac{\partial v_{i}}{\partial x_{j}}+\frac{\partial v_{j}}{\partial x_{i}}\right)=0, D_{i i}=D_{12}=0, i, j=1,2,3,
$$

the boundary condition $\left(t=\tau^{*}\left(x_{1}, x_{2}\right)\right)$

$$
\left(x_{1}, x_{2}\right) \in L^{*}(t):\left\{\begin{array}{l}
\sigma_{13}=\sigma_{13}^{*}, \quad \sigma_{23}=\sigma_{23}^{*}, \\
\frac{\partial \sigma_{13}}{\partial t} n_{1}+\frac{\partial \sigma_{23}}{\partial t} n_{2}=0
\end{array}\right.
$$

on the growth boundary, the initial-boundary condition

$$
\left(x_{1}, x_{2}\right) \in L_{\sigma}: \sigma_{13} n_{1}+\sigma_{23} n_{2}=0
$$

on the immovable boundary, and the constitutive equations

$$
\begin{gathered}
\sigma_{13}=2 G\left(I+N\left(\tau_{0}\left(x_{1}, x_{2}\right), t\right)\right) \varepsilon_{13}, \\
\sigma_{23}=2 G\left(I+N\left(\tau_{0}\left(x_{1}, x_{2}\right), t\right)\right) \varepsilon_{23}, \\
\tau_{0}^{*}\left(x_{1}, x_{2}\right)=\left\{\begin{array}{c}
\tau_{0},\left(x_{1}, x_{2}\right) \in \Omega_{1}, \\
\tau^{*}\left(x_{1}, x_{2}\right),\left(x_{1}, x_{2}\right) \in \Omega^{*}(t),
\end{array}\right.
\end{gathered}
$$

where $\Omega^{*}(t)=\Omega(t) \backslash \Omega_{1}$, the operator $\left(I-L\left(\tau_{0}^{*}, t\right)\right)$ and the inverse operator $\left(I+N\left(\tau_{0}^{*} t\right)\right)$ can be determined from (4) by the replacement of $\tau_{0}$ with $\tau_{0}^{*}$, and $\sigma_{13}^{*}\left(x_{1}, x_{2}, \tau^{*}\right)$ and $\sigma_{23}^{*}\left(x_{1}, x_{2}, \tau^{*}\right)$ are the components of the 2D stress tensor acting on the surface accreted by the 3D body.

The equilibrium conditions for the end cross-section $\Omega(t)$ subjected to the torque $M(t)$ have the form

$$
M(t)=\iint_{\Omega(t)}\left(x_{1} \sigma_{23}-x_{2} \sigma_{13}\right) d x_{1} d x_{2} .
$$

Relations (32)-(37) form the general boundary value problem for a continuously growing body. Relations (36) show that in general the process of accretion of new elements leads to constitutive relations with discontinuities on the interface between the main body and the additional bodies. 
Let us transform the initial-boundary value problem for a continuously growing viscoelastic ageing body into a problem with time parameter coinciding in form with a boundary value problem of the elasticity theory. At the first stage, we transform the accretion problem for a viscoelastic body with constitutive relations (36) to the accretion problem for an elastic body described by Hooke's law.

To this end, we represent the equation of the growth boundary $L^{*}(t)$ for $t=\tau_{1}$ in the form

$$
\left(x_{1}, x_{2}\right) \in L^{*}(t): \quad \tau_{1}-\tau^{*}=0,
$$

where $\tau_{1}-\tau^{*} \geq 0$ for $\left(x_{1}, x_{2}\right) \in \Omega_{0}$ and $\tau_{1}-\tau^{*}<0$ for $\left(x_{1}, x_{2}\right) \in \Omega^{*}(t)$. We assume that $\tau^{*}$ is a sufficiently smooth function such that $\nabla \tau^{*} \neq 0$ for $\tau^{*}-\tau_{1}=0$. (That is, there are no singular points on the growth boundary.) We introduce the characteristic function $\theta\left(\tau_{1}-\tau^{*}\right)$, which is equal to unity if the argument of the function is nonnegative and is zero if the argument is negative. Now the operator $\left(I-L\left(\tau_{0}^{*}, t\right)\right)$ can be represented in the form

$$
\begin{gathered}
\left(I-L\left(\tau_{0}^{*}, t\right)\right) f(t)=\left(I-L\left(\tau^{0}, t\right)\right) f(t)-\theta\left(\tau_{1}-\tau^{*}\right) L^{t}\left(\tau_{0}, \tau_{1}\right) f(t) \\
L^{t}\left(\tau_{0}, \tau_{1}\right) f(t)=\int_{\tau_{0}}^{\tau_{1}} f(\tau) K_{1}(t, \tau) d \tau, \\
\tau^{0}=\theta\left(\tau_{1}-\tau^{*}\right)\left(\tau_{1}-\tau^{*}\left(x_{1}, x_{2}\right)\right)+\tau^{*}\left(x_{1}, x_{2}\right),
\end{gathered}
$$

where $\tau^{*}=\tau_{1}$ for $\left(x_{1}, x_{2}\right) \in L^{*}\left(\tau_{1}\right)$.

Let us divide the relations containing the stresses $\sigma_{13}$ and $\sigma_{23}$ in (32)-(37) by $G$ and then apply the operator $\left(I-L\left(\tau_{0}^{*}, t\right)\right.$. Then, in view of the notation $\sigma_{i j}^{0}=\left(I-L\left(\tau_{0}^{*}, t\right)\right) \sigma_{i j} G^{-1}$, we obtain

$$
\begin{gathered}
\frac{\partial \sigma_{13}^{0}}{\partial x_{3}}=0, \quad \frac{\partial \sigma_{23}^{0}}{\partial x_{3}}=0, \quad \frac{\partial \sigma_{13}^{0}}{\partial x_{1}}+\frac{\partial \sigma_{23}^{0}}{\partial x_{2}}=0, \\
D_{i j}=\frac{1}{2}\left(\frac{\partial v_{i}}{\partial x_{j}}+\frac{\partial v_{j}}{\partial x_{i}}\right)=0, D_{i i}=D_{12}=0, i, j=1,2,3, \\
\sigma_{13}^{0}=2 \varepsilon_{13}, \quad \sigma_{23}^{0}=2 \varepsilon_{23}, \\
\left(x_{1}, x_{2}\right) \in L^{*}(t):\left\{\begin{array}{c}
\sigma_{13}^{0}=\sigma_{13}^{*} G^{-1}, \sigma_{23}^{0}=\sigma_{23}^{*} G^{-1}, \\
\frac{\partial \sigma_{13}^{0}}{\partial t} n_{1}+\frac{\partial \sigma_{23}^{0}}{\partial t} n_{2}=0 ; \\
\left(x_{1}, x_{2}\right) \in L_{\sigma}: \quad \sigma_{13}^{0} n_{1}+\sigma_{23}^{0} n_{2}=0, \\
M^{0}(t)=\iint_{\Omega(t)}\left(x_{1} \sigma_{23}^{0}-x_{2} \sigma_{13}^{0}\right) d x_{1} d x_{2} .
\end{array}\right.
\end{gathered}
$$

Let us transform the initial-boundary value problem (40) to a boundary value problem for the strain rates, the displacement rates, and the operator stress rates. To this end, we differentiate the equilibrium equations, the constitutive equations, the boundary condition on the immovable boundary $L_{\sigma}(t)$, and the equilibrium condition for the end cross-section with respect to $t$ and obtain the boundary value problem

$$
\begin{gathered}
\frac{\partial S_{13}}{\partial x_{3}}=0, \quad \frac{\partial S_{23}}{\partial x_{3}}=0, \quad \frac{\partial S_{13}}{\partial x_{1}}+\frac{\partial S_{23}}{\partial x_{2}}=0 \\
D_{i j}=\frac{1}{2}\left(\frac{\partial v_{i}}{\partial x_{j}}+\frac{\partial v_{j}}{\partial x_{i}}\right)=0, D_{i i}=D_{12}=0, i, j=1,2,3, \\
S_{13}=2 D_{13}, \quad S_{23}=2 D_{23}, \\
\left(x_{1}, x_{2}\right) \in L^{*}(t): \quad S_{13} n_{1}+S_{13} n_{2}=0 \\
\left(x_{1}, x_{2}\right) \in L_{\sigma}: \quad S_{13} n_{1}+S_{13} n_{2}=0 ; \\
M^{0 \bullet}(t)=\iint_{\Omega(t)}\left(x_{1} S_{23}-x_{2} S_{13}\right) d x_{1} d x_{2}+\int_{L^{*}(t)}\left(x_{1} \sigma_{23}^{0}-x_{2} \sigma_{13}^{0}\right) S_{n} d l,
\end{gathered}
$$

where the second term in the last formula is a contour integral over the boundary $L^{*}(t)$.

Being supplemented with the initial conditions for the main body at $t=\tau_{1}$, relations (40), which also contain the initial-boundary condition on the growth boundary, form an initial-boundary value problem with time parameter $t$.

The formulas for the variables $v_{i}$ and $S_{i j}$ can be obtained by the replacement of the function $\varphi\left(x_{1}, x_{2}, \tau_{1}\right)=\varphi_{\tau_{1}}$ by $\varphi\left(x_{1}, x_{2}, t\right)=\varphi_{t}$ :

$$
\begin{aligned}
& v_{1}=-\theta^{\bullet}(t) x_{2} x_{3}, v_{2}=\theta^{\bullet}(t) x_{3} x_{1}, v_{3}=\theta^{\bullet}(t) \varphi_{t}, \\
& S_{13}=\theta^{\bullet}(t)\left(\frac{\partial \varphi_{t}}{\partial x_{1}}-x_{2}\right), S_{23}=\theta^{\bullet}(t)\left(\frac{\partial \varphi_{t}}{\partial x_{2}}+x_{1}\right)
\end{aligned}
$$

The torsion function $\varphi_{t}$ can be found from the Neumann boundary value problem

$$
\begin{aligned}
& \frac{\partial^{2} \varphi_{t}}{\partial x_{1}^{2}}+\frac{\partial^{2} \varphi_{t}}{\partial x_{2}^{2}}=0, \\
& \left(x_{1}, x_{2}\right) \in L^{*}(t): \quad \frac{\partial \varphi_{t}}{\partial n}=x_{2} n_{1}-x_{1} n_{2} .
\end{aligned}
$$

Thus, we have reduced the original torsion problem for a prismatic body to the Neumann problem for the function $\varphi_{t}$ in the domain $\Omega(t)$.

Obviously, in view of (42) we obtain

$$
\begin{aligned}
& M^{0 \bullet}(t)=\theta^{\bullet}(t) D_{t}+\int_{L^{*}(t)}\left(x_{1} \sigma_{23}^{0}-x_{2} \sigma_{13}^{0}\right) s_{n} d l, \\
& D_{t}=\iint_{\Omega(t)}\left(x_{1}^{2}+x_{2}^{2}+x_{1} \frac{\partial \varphi_{t}}{\partial x_{2}}-x_{2} \frac{\partial \varphi_{t}}{\partial x_{1}}\right) d x_{1} d x_{2},
\end{aligned}
$$

where $D_{t}$ is the variable torsional rigidity of the growing body and $\varphi_{t}$ is the unknown harmonic function.

At the stage of growth of the twisted body, we also have two possible versions of the statement of the problem.

To solve the first version (where the torque $M(t)$ is given), one should use the boundary condition to find $\varphi_{t}$, then determine $v_{i}$ and $S_{i j}$ by formulas (42), use formulas (44) to find the torsion angular velocity, and reconstruct the true stresses, displacements, and torsion angle by the formulas 


$$
\begin{gathered}
\sigma_{i j}\left(x_{1}, x_{2}, t\right)=G(t)\left[\frac{\sigma_{i j}\left(x_{1}, x_{2}, \tau_{0}^{*}\right)}{G\left(\tau_{0}^{*}\right)}\left(1+\int_{\tau_{0}^{*}}^{t} R(t, \tau) d \tau\right)+\right. \\
\left.+\int_{\tau_{0}^{*}}^{t}\left(S_{i j}\left(x_{1}, x_{2}, \tau\right)+\int_{\tau_{0}^{*}}^{\tau} S_{i j}\left(x_{1}, x_{2}, \varsigma\right) d \varsigma R(t, \tau)\right) d \tau\right] \\
u_{i}\left(x_{1}, x_{2}, t\right)=u_{i}\left(x_{1}, x_{2}, \tau_{0}^{*}\right)+\int_{\tau_{0}}^{t} *_{i}\left(x_{1}, x_{2}, \tau\right) d \tau, \\
\theta(t)=\theta\left(\tau_{0}^{*}\right)+\int_{\tau_{0}^{*}}^{t} \theta \cdot(\tau) d \tau .
\end{gathered}
$$

To solve the second version of the problem (where the torsion angle $\theta(t)$ is given), one should find $\varphi_{t}$, determine $v_{i}$ and $S_{i j}$ by formulas (42), reconstruct $u_{i}$ and $\sigma_{i j}$ by formulas (45), and find the torque according to formula (5).

To solve the boundary value problem (43), one can apply methods of the theory of functions of one complex variable, which we will discuss below.

Thus, the study of the torsion problem for a growing body at the stage of continuous accretion is complete.

\section{Deformation of the rod after the termination of growth}

Let accretion terminate at time $\tau_{2}$. At this time, the body occupies the domain $\Pi_{2}$ whose cross-section $\Omega_{2}$ is bounded by the contour $L_{2}$. On the time interval $t \in\left[\tau_{2}, \tau_{3}\right]$ (where $\tau_{3}$ is the time at which the next accretion stage begins), the problem for the nonvarying body is similar to problem (32)-(36), where the initialboundary condition on the growth boundary $L^{*}(t)$ is lacking,

$$
\begin{gathered}
\frac{\partial \sigma_{13}}{\partial x_{3}}=0, \quad \frac{\partial \sigma_{23}}{\partial x_{3}}=0, \quad \frac{\partial \sigma_{13}}{\partial x_{1}}+\frac{\partial \sigma_{23}}{\partial x_{2}}=0, \\
D_{i j}=\frac{1}{2}\left(\frac{\partial v_{i}}{\partial x_{j}}+\frac{\partial v_{j}}{\partial x_{i}}\right)=0, D_{i i}=D_{12}=0, i, j=1,2,3, \\
\sigma_{13}=2 G\left(I+N\left(\tau_{0}^{*}, t\right) \varepsilon_{13}, \quad \sigma_{23}=2 G\left(I+N\left(\tau_{0}^{*}, t\right) \varepsilon_{23},\right.\right. \\
\left(x_{1}, x_{2}\right) \in L_{2}: \quad \sigma_{13} n_{1}+\sigma_{23} n_{2}=0, \\
M(t)=\iint_{\Omega_{2}}\left(x_{1} \sigma_{23}-x_{2} \sigma_{13}\right) d x_{1} d x_{2} .
\end{gathered}
$$

By analogy with the preceding, we obtain the boundary value problem

$$
\begin{gathered}
\frac{\partial S_{13}}{\partial x_{3}}=0, \quad \frac{\partial S_{23}}{\partial x_{3}}=0, \quad \frac{\partial S_{13}}{\partial x_{1}}+\frac{\partial S_{23}}{\partial x_{2}}=0, S_{13}=2 D_{13}, \quad S_{23}=2 D_{23}, \\
D_{i j}=\frac{1}{2}\left(\frac{\partial v_{i}}{\partial x_{j}}+\frac{\partial v_{j}}{\partial x_{i}}\right)=0, D_{i i}=D_{12}=0, i, j=1,2,3, \\
\left(x_{1}, x_{2}\right) \in L_{2}: \quad S_{13} n_{1}+S_{13} n_{2}=0 ; \\
M^{0 \bullet}(t)=\iint_{\Omega_{2}}\left(x_{1} S_{23}-x_{2} S_{13}\right) \ldots d x_{1} d x_{2}+\int_{L_{2}}\left(x_{1} \sigma_{23}^{0}-x_{2} \sigma_{13}^{0}\right) S_{n} d l .
\end{gathered}
$$

Problem (47) is obviously similar to problem (26). In this case, formulas (27), where the function $\varphi_{\tau_{1}}$ should be replaced by $\varphi_{\tau_{2}}=\varphi\left(x_{1}, x_{2}, \tau_{2}\right)$, apply to the variables $v_{i}$ and $S_{i j}$,

$$
\begin{aligned}
& v_{1}=-\theta^{\bullet}(t) x_{2} x_{3}, v_{2}=\theta^{\bullet}(t) x_{3} x_{1}, v_{3}=\theta^{\bullet}(t) \varphi_{\tau_{2}}, \\
& S_{13}=\theta^{\bullet}(t)\left(\frac{\partial \varphi_{\tau_{2}}}{\partial x_{1}}-x_{2}\right), S_{23}=\theta^{\bullet}(t)\left(\frac{\partial \varphi_{\tau_{2}}}{\partial x_{2}}+x_{1}\right) .
\end{aligned}
$$

To find the function $\varphi_{\tau_{2}}$, it suffices to solve the Neumann problem

$$
\begin{gathered}
\frac{\partial^{2} \varphi_{\tau_{2}}}{\partial x_{1}^{2}}+\frac{\partial^{2} \varphi_{\tau_{2}}}{\partial x_{2}^{2}}=0,\left(x_{1}, x_{2}\right) \in L_{2}: \frac{\partial \varphi_{\tau_{2}}}{\partial n}=x_{2} n_{1}-x_{1} n_{2}, \\
M^{0 \bullet}(t)=\theta^{\bullet}(t) D_{2}+\int_{L_{2}}\left(x_{1} \sigma_{23}^{0}-x_{2} \sigma_{13}^{0}\right) s_{n} d l, \\
D_{2}=\iint_{\Omega_{2}}\left(x_{1}^{2}+x_{2}^{2}+x_{1} \frac{\partial \varphi_{\tau_{2}}}{\partial x_{2}}-x_{2} \frac{\partial \varphi_{\tau_{2}}}{\partial x_{1}}\right) d x_{1} d x_{2} .
\end{gathered}
$$

To find the solution of the resulting Neumann boundary value problems, one can use methods of complex analysis, which are presented, say, in [9]. The true stresses and displacements can be completely reconstructed with the use of the decoding formulas (45).

\section{Conclusions}

- A torsion theory of growing viscoelastic prismatic rods is constructed. Statements of the corresponding classical and nonclassical initial-boundary value problems are given.

- Solution methods for such problems are suggested based on the reduction on nonclassical problems for growing bodies to elasticity problems with a parameter. The subsequent use of complex analysis and the decoding formulas developed in the paper permits one to reconstruct the true values of stressstrain characteristics of growing rods.

- A new effect in mechanics is revealed. In the classical case, the maximum tangential stress intensity is known to be attained on the boundary of the body. In the case of a growing rod, the maximum tangential stress intensity can be attained at an arbitrary point of the grown part depending on the growth mode.

- The results obtained can serve as a basis for modeling the processes of crystal growth from melt, manufacturing or repairing machine parts by spraying, weld overlay cladding, or precipitation, and a number of other additive manufacturing processes (see also [10-16]).

\section{Acknowledgements}

This work was financially supported by the Russian Science Foundation under Grant 14-19-01280.

\section{References}

1. N. Kh. Arutyunyan, A. V. Manzhirov, and V. E. Naumov, Contact Problems in Mechanics of Growing Bodies [in Russian] (Nauka Publ., Moscow,1991) 
2. A. V. Manzhirov, Journal of Applied Mathematics and Mechanics 59 (5), 805-816 (1995)

3. N. Kh. Arutyunyan and A. V Manzhirov, Contact Problems in the Theory of Creep [in Russian] (NAS Armenia, Yerevan,1999)

4. A. V. Manzhirov, Key Engineering Materials, 535536, 89-93 (2013) doi: 10.4028/www.scientific.net/ KEM.535-536.89

5. A. V. Manzhirov, S.A. Lychev, and N. K. Gupta, Proc. Indian Natl Sci Acad. 79 (4), 529-545 (2013) doi: 10.16943/ptinsa/2013/v79i4/47989

6. A. V. Manzhirov, Proceedings of ASME 2014 International Mechanical Engineering Congress \& Exposition, Volume 9: Mechanics of Solids, Structures and Fluids (Montreal, Quebec, Canada) 10 p. (2014) doi:10.1115/IMECE2014-36712

7. A. Manzhirov and N. Gupta, IUTAM Symposium on Growing solids. Symposium Materials (In-t Probl. Mech. RAS, Moscow, 2015) 73-76

8. N. Kh. Arutyunyan and B. L. Abramyan, Torsion of Elastic Solids [in Russian] (Fizmatgiz, Moscow, 1963)

9. N. I. Muskhelishvili, Some Basic Problems of the Mathematical Theory of Elasticity (Springer, Netherlands, 2010)
10. A. V. Manzhirov and S. A. Lychev, Lecture Notes in Engineering and Computer Science: World Congress on Engineering (London, IAENG, 2014) 1404-1409

11. S. A. Lychev and A. V. Manzhirov, Lecture Notes in Engineering and Computer Science: World Congress on Engineering (London, IAENG, 2014) 1327-1332

12. A. V. Manzhirov and S. A. Lychev, Transactions on Engineering Technologies: World Congress on Engineering 2014 (Springer, Netherlands, 2015) 99115 doi: 10.1007/978-94-017-9804-4

13. S. A. Lychev, A. V. Manzhirov, and P. S. Bychkov, Transactions on Engineering Technologies: World Congress on Engineering 2014 (Springer, Netherlands, 2015) 239-254 doi: 10.1007/978-94-017-9804-4

14. A. V. Manzhirov and D. A. Parshin, Mechanics of Solids. 50 (5), 559-570 (2015) doi: 10.3103/ S0025654415050076

15. A. V. Manzhirov and D. A. Parshin, Mechanics of Solids. 50 (6), 657-675 (2015) doi: 10.3103/ S0025654415060072

16. A. V. Manzhirov, Lecture Notes in Engineering and Computer Science: Proceedings of the World Congress on Engineering 2015 (London, IAENG, 2015) 710-714 